\title{
GELYELLA DROGUEI N. G., N. SP., CURIEUX HARPACTICIDE DES EAUX SOUTERRAINES CONTINENTALES DE LA NOUVELLE faMille DES GeLYelLidAe
}

\author{
par R. ROUCH et F. LESCHER-MOUTOUÉ'.
}

Description d'une nouvelle espèce découverte dans les eaux souterraines karstiques continentales de la région de Montpellier (France). De nombreux caractères originaux tels la structure primitive des appendices céphaliques, la disparition de la P4 et de la P5, la réduction de la segmentation des exopodites et des endopodites des $\mathrm{P} 1$ à $\mathrm{P} 3$, nous ont conduits à créer une famille et un genre nouveaux.

\section{Gelyella droguei n. g., n. sp., an interesting harpacticoid from continental subterranean waters of the new family Gelyellidae.}

A new species collected in the subterranean water of a karstic system near Montpellier ((France), is described. Numerous original characters, such as primitive structure of mouthparts, lack of $\mathbf{P 4}$ and $(\mathrm{P5}$, reduction of segmentation in swimming legs, place this species in a new genus and family.

L'étude des eaux douces souterraines du Languedoc a conduit l'un d'entre nous (F. Lescher-Moutoué) à capturer un Copépode très remarquable par l'ensemble de ses caractères monphologiques.

Les récoltes ont été effectuées au cours de pompages d'essais réalisés du 18 au 20 mars 1976 par le Laboratoire d'Hydrogéologie de l'Université des Sciences et Techniques du Languedoc à Montpellier. Vingt heures de filtrage, pour un débit moyen égal à $110 \mathrm{~m}^{3} / \mathrm{h}$, ont permis la capture de 22 individus ( 9 femelles, 10 mâles et 3 copépodites).

Le forage, profond de soixante mètres, est situé sur le territoire de la commune de Saint-Gély-du-Fesc, à douze kilomètres au nordouest de Montpellier (Hérault, France). Implanté sur la partie est monoclinale du synclinal de Saint-Gély-du-Fesc, ce forage atteint des calcaires lacustres d'âge lutétien (Eocène) inclus entre des marnes infra-lutétiennes et oligocènes; leur puissance varie de quatre-vingts à cent mètres. Une telle disposition a favorisé l'accumulation de réserves d'eau importantes dans ces roches carbonatées qui sont karstifiées en profondeur en raison de conditions géomorphologiques très localisées. En surface, au contraire, les phénomènes de karstification sont extrêmement rares.

1. Laboratoire souterrain du C.N.R.S., 09410 Moulis, France. 


\section{1. - GELYELLA DROGUEI N. G., N. SP. ${ }^{2}$}

\section{Femelle}

La taille de quatre individus mesurés est comprise entre 308 et $327 \mu \mathrm{m}$. En vue dorsale, les bords du corps sont sub-parallèles sans qu'apparaisse une distinction nette entre le thorax et l'abdomen. Le rostre, dont l'extrémité distale est arrondie, ne dépasse pas le premier tiers de la longueur de l'article proximal de l'antennule. Le premier segment thoracique n'étant pas soudé avec la tête et la fusion des deux premiers segments abdominaux en un segment génital étant parfaitement réalisée, le nombre total des segments du corps est égal à dix ; leur bordure postérieure est lisse, dorsalement et ventralement. L'aire génitale, sclérifiée, présente une structure relativement simple (voir fig. 4). Le dernier segment abdominal est remarquablement long; l'opercule anal, de forme arrondie, ne
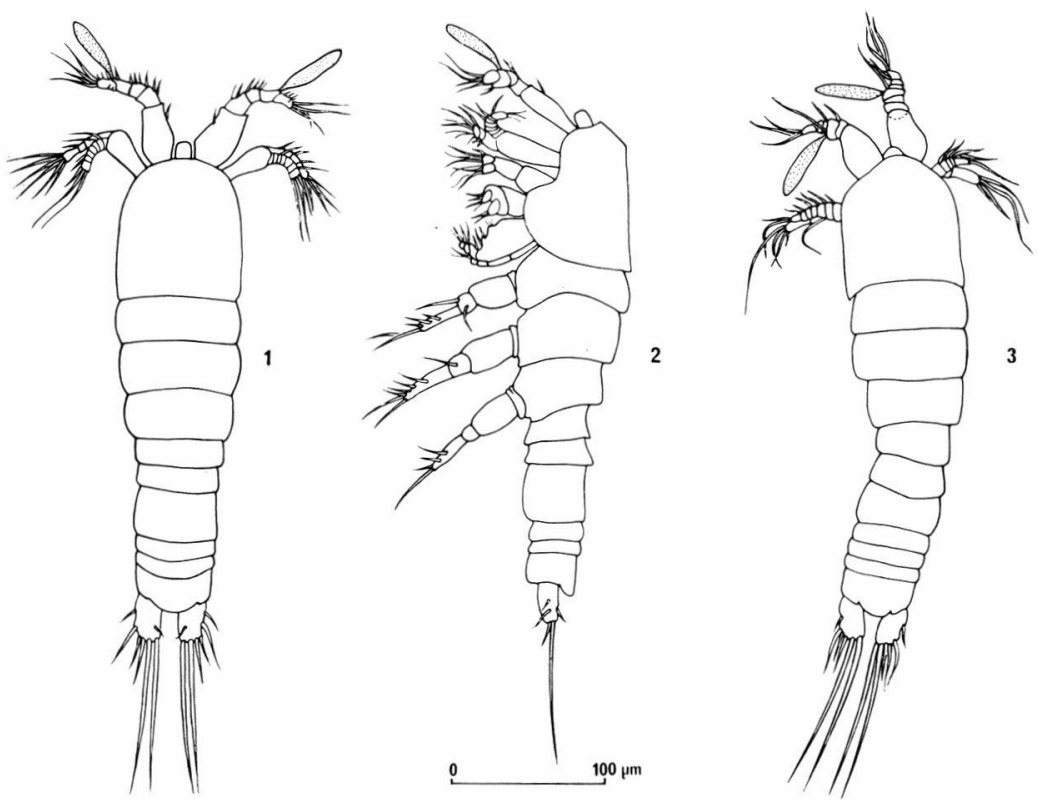

FIG. 1 à 3. - Gelyella droguei n. g., n. sp. - Fig. 1: habitus de la femelle en vue dorsale. - Fig. 2: habitus de la femelle en vue latérale. - Fig. 3 : habitus du mâle en vue dorsale.

2. Le nom générique fait référence à la localité de Saint-Gély-du-Fesc, où cette espèce a été découverte grâce à l'aide de C. Drogue à qui nous sommes heureux de la dédier. 
porte aucune ornementation. Les branches furcales, quadrangulaires, sont deux fois plus longues que larges. Elles présentent une épine et deux soies sur le bord externe, deux à trois soies assez fines insérées ventralement sur le bord interne, une soie dorsale et quatre soies apicales; parmi ces dernières, seules les deux internes sont très développées.

Antennule. - Elle apparaît composée de cinq articles, le premier étant aussi long que tous les autres réunis. Le quatrième article porte un aesthète en forme de palette à la base duquel s'insère une longue soie. Le cinquième article présente à sa base deux replis chitineux qui pourraient être interprétés comme des limites de segment. La partie distale du second article offrant le même type de différenciation, la segmentation de l'antennule s'avère donc assez indistincte. Mais c'est là un caractère qui a été quelquefois signalé

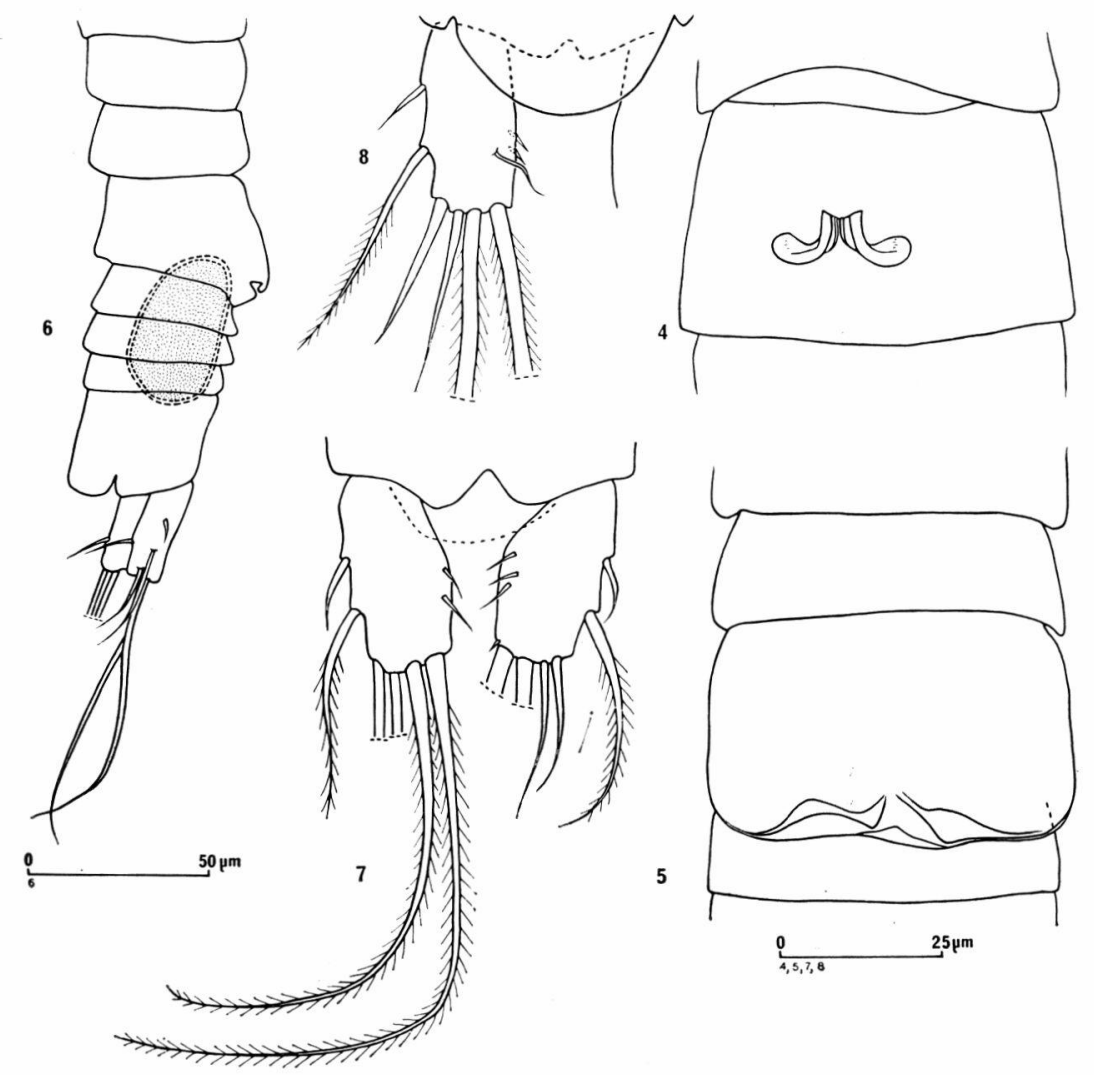

FIG. 4 à 8. - Gelyella droguei n. g., n. sp. - Fig. 4 : segment génital femelle. Fig. 5: segment génital mâle. - Fig. 6: abdomen du mâle en vue latérale. Fig. 7 : branches furcales et opercule anal du mâle. - Fig. 8: branches furcales de la femelle. 
chez les Harpacticides, notamment chez les Canuellidae (Coull 1971, 1972 ; Bozic 1968 ; Hamond 1975).

Antenne. - Coxa glabre. Basis avec deux petites soies sur le bord interne. L'endopodite est formé de trois articles dont le distal est orné de sept soies. L'exopodite est composé de sept articles; les six premiers portent chacun une longue soie à leur angle distal et interne, le dernier trois soies.

Mandibule. - Le basis du palpe mandibulaire est armé d'une seule soie. L'exopodite, à segmentation peu distincte, est formé de trois articles ornés respectivement de une, deux et trois soies. L'endopodite, biarticulé, possède trois soies sur le premier article et huit soies sur le second.

MaXILlule. - L'arthrite précoxal porte six soies et épines. La coxa est armée d'une soie et d'une épine mais ne présente aucun épipodite. Le basis est orné de six soies. L'endopodite et l'exopodite sont uniarticulés; le premier est armé de six soies, le second de trois.
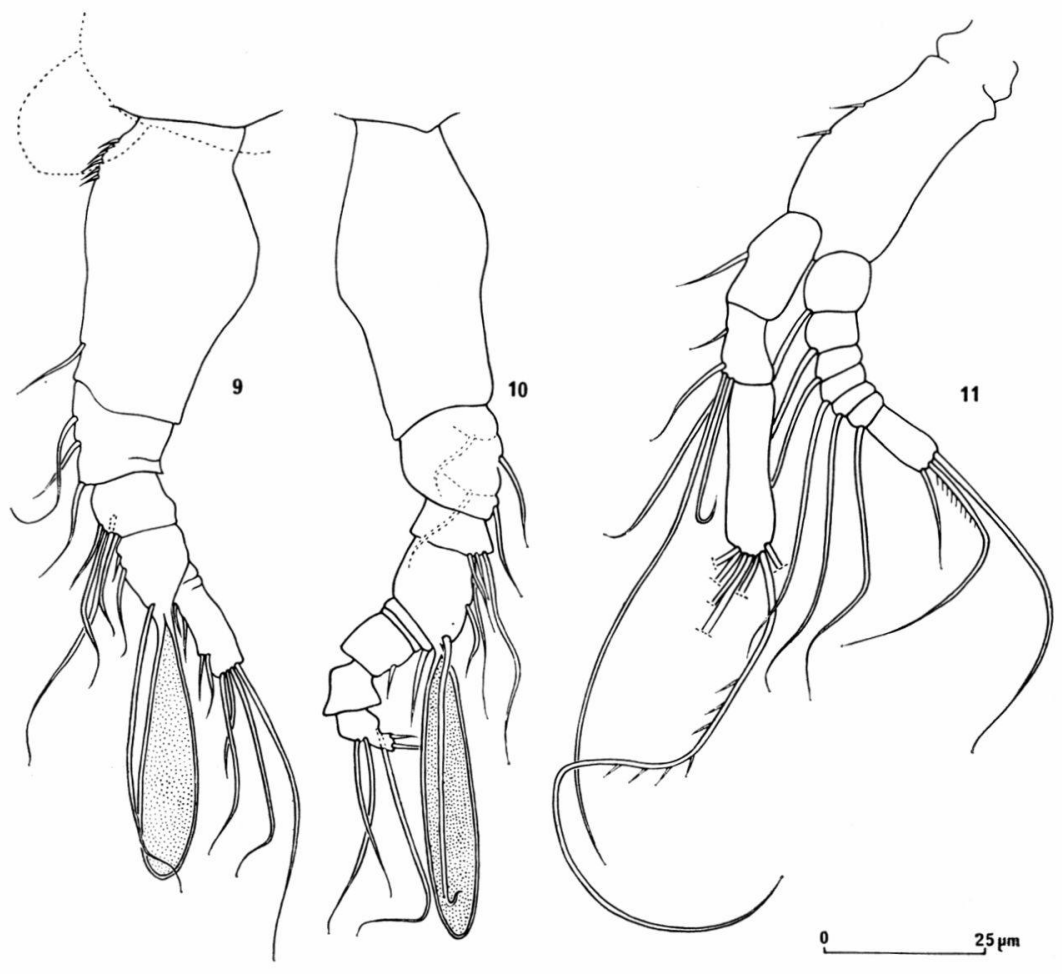

Fig. 9 à 11. - Gelyella droguei n. g., n. sp. - Fig. 9: A1 de la femelle. - Fig. 10: A1 du mâle. - Fig. 11: A2 de la femelle. 


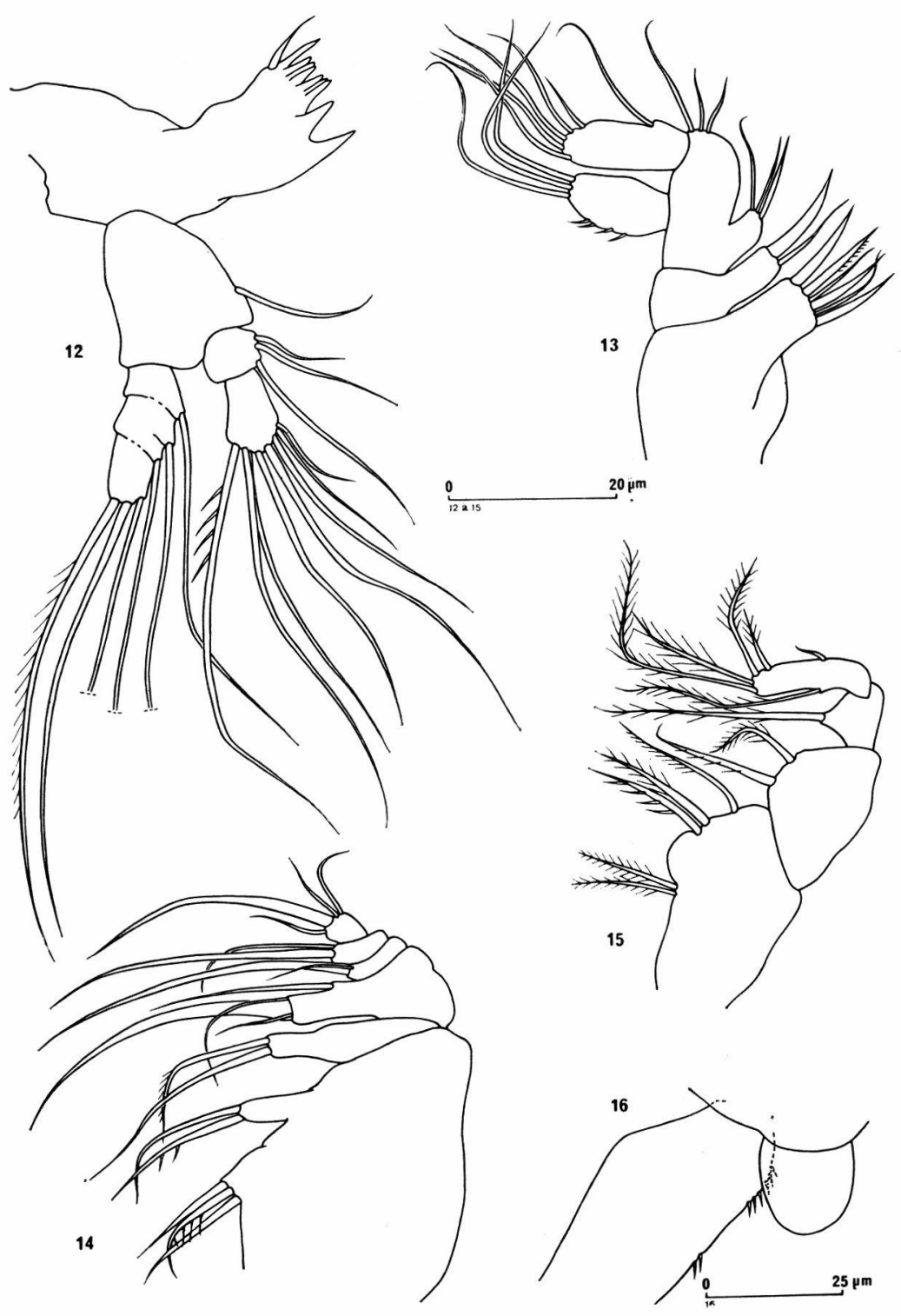

Fig. 12 à 16. - Gelyella droguei n. g., n. sp. femelle. - Fig. 12: mandibule. Fig. 13 : maxillule. - Fig. 14 : maxille. - Fig. 15 : maxillipède. - Fig. 16 : rostre. 
Maxille. - La précoxa porte deux endites armés respectivement de trois et deux soies. La coxa présente un seul endite orné de deux soies apicales. Le basis offre un seul endite avec une soie latérale et une forte épine apicale flanquée, à sa base, de deux petites soies. L'endopodite est triarticulé; les deux premiers articles sont ornés d'une épine et d'une soie, le troisième possède une épine et trois soies.

Maxillipède. - Il est composé de quatre éléments, un coxopodite, un basis et un endopodite biarticulé ornés respectivement de cinq, deux, une et six soies.

Pattes thoracioues. - Gelyella droguei est caractérisée par l'absence non seulement de la P5, mais également de la P4 dont on ne retrouve absolument aucun vestige. Cette disparition des deux dernières paires de pattes thoraciques constitue un des caractères étonnants de cette forme.

Les trois paires de pattes natatoires sont démunies de soie au bord interne et distal des coxa. En revanche, les basis de P1 et de P2 sont ornés d'une épine sur leur bord externe; cette épine fait complètement défaut sur la P3.

Les exopodites de P1, P2 et P3 ne montrent aucune trace de segmentation. Ils sont ornés respectivement de six, sept et six soies et épines.

L'endopodite P1 est réduit à une épine assez mince s'insérant directement sur le basis et atteignant la moitié de l'exopodite.

Les endopodties P2 et P3 se présentent sous forme d'une petite protubérance portant à son extrémité distale une longue soie.

Les appendices sont donc très réduits tant au niveau de leur nombre que de leur segmentation et de leur configuration générale.

\section{Mâle}

La taille des cinq individus mesurés est comprise entre 305 et $344 \mu \mathrm{m}$. En l'absence de fusion du premier segment thoracique avec la tête, le mâle possède au total onze segments. L'ornementation du corps, la forme de l'opercule anal et des branches ifurcales ne diffèrent pas de ce qui a été observé chez la femelle. La partie distale du segment génital présente deux bourrelets chitineux situés sur la bordure distale.

L'antennule est haplocère et très indistinctement segmentée.

L'antenne et les pièces buccales ne diffèrent pas de celles de la femelle.

Comme chez celle-ci, il n'existe aucune trace de la P4 et de la P5. 


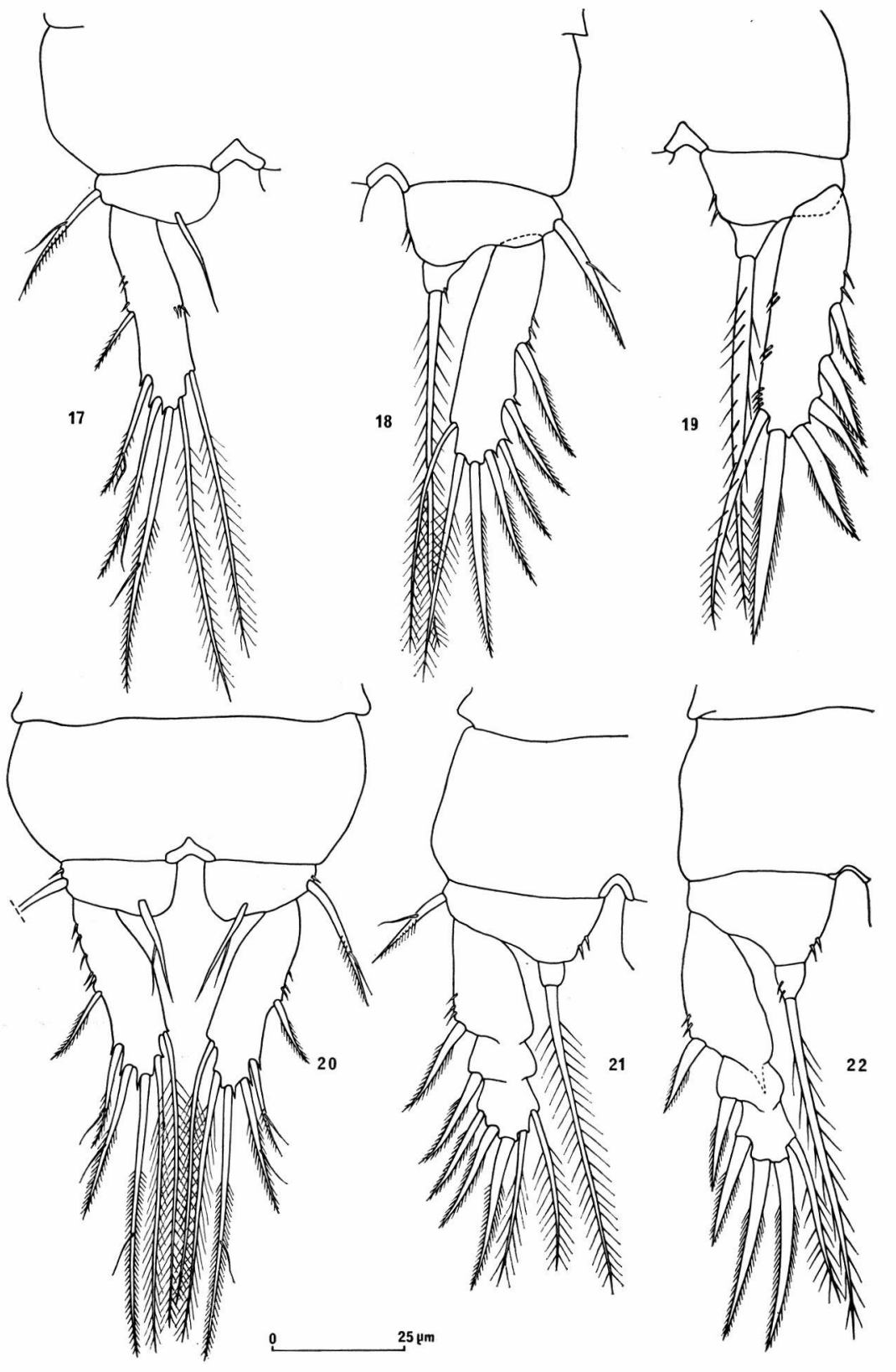

FIG. 17 à 22. - Gelyella droguei n. g., n. sp. - Fig. 17 : P1 de la femelle. - Fig. 18: P2 de la femelle. - Fig. 19: P3 de la femelle. - Fig. 20: P1 du mâle. Fig. 21 : P2 du mâle. - Fig. 22: P3 du mâle. 
La P1 du mâle est semblable à celle de la femelle avec six épines insérées sur l'exopodite uniarticulé et un endopodite réduit à une simple soie. La P2 présente des traces de segmentation sur l'exopodite ; l'ornementation de ce dernier est identique à celle de la femelle. L'endopodite est inchangé par rapport à celui de l'autre sexe et ne présente donc aucun caractère sexuel secondaire. La P3 offre également des traces de segmentation sur l'exopodite qui porte six addendes; l'endopodite ne diffère pas de celui de la femelle.

Chez le mâle également, aucune des trois paires de pattes n'est ornée d'une épine au bord interne et distal des coxa et seules la P1 et la $\mathrm{P} 2$ possèdent une épine au bord externe du basis.

\section{2. - DISCUSSION}

L'espèce de Saint-Gély-du-Fesc est difficile à classer en raison de ses caractères morphologiques. La forme allongée de son corps, sans limite nette entre le thorax et l'abdomen, le nombre réduit des articles de l'antennule permettent d'affirmer qu'il s'agit là d'un Harpacticide.

Toute hypothèse sur des relations phylétiques doit être basée sur des synapomorphies (caractères évolués communs, Hennig 1965). Avec une rigueur assez rare pour son époque, Lang (1948) a distingué les stades primitifs et les stades évolués des structures qu'il utilisait: aussi est-il possible de rechercher quelle position occupe Gelyella dans le système qu'il a proposé.

Lang divise les Harpacticides en Polyarthra et en Oligoarthra. Les deux familles des Polyarthra, les Longipediidae et les Canuellidae, ne présentent pas de synapomorphie; aussi n'est-il pas exclu que les Polyarthra soient paraphylétiques. Les Oligoarthra sont caractérisés par les synapomorphies suivantes: l'antenne possède un exopodite avec 4 articles au plus et un endopodite tout au plus biarticulé. L'endopodite de la mandibule est au plus uniarticulé. Les coxa des P1 n'ont pas de soie interne.

Discutons maintenant les structures de Gelyella droguei.

La disparition de la $\mathrm{P} 4$ et de la $\mathrm{P} 5$, la réduction extrême des trois paires de pattes natatoires chez la femelle et le mâle constituent autant de caractères qui, en première analyse, n'apportent guère d'éléments positifs dans la recherche de l'ensemble auquel appartient cette espèce. Il faut néanmoins noter au niveau des pattes du mâle l'absence de différenciation en organe aidant à l'accouplement car la segmentation peu apparente des exopodites ne peut être considérée comme telle. 
L'étude des appendices céphaliques est beaucoup plus riche d'enseignements.

Si l'antennule ne fournit à ce niveau des investigations que fort peu d'éléments, encore que le nombre d'articles qui la composent soit réduit, il n'en est pas de même pour l'antenne. La structure de celleci est extrêmement nette; avec sept articles à l'exopodite et trois articles à l'endopodite, l'antenne est bâtie sur le type en vigueur dans la section des Polyarthra, notamment chez les Canuellidae. Chez les Oligoarthra au contraire, la structure de l'A2 est beaucoup moins primitive, l'endopodite étant tout au plus diarticulé et l'exopodite quadriarticulé. La mandibule dont le palpe porte un exopodite triarticulé et un endopodite biarticulé est également conforme à ce que l'on connaît chez les Polyarthra; elle diffère de celle des Oligoarthra dont l'endopodite est uniarticulé. La maxillule de Gelyella bien que bâtie selon un type primitif ne présente aucune trace d'un épipodite sur la coxa ce qui est toujours le cas chez les Polyarthra; en outre l'endopodite de cet appendice est uniarticulé alors qu'il est biarticulé chez la plupart des Polyarthra; Hamond (1973) signale toutefois un endopodite uniarticulé chez certaines espèces de Brianola (Canuellidae). La maxille avec une précoxa, une coxa, un basis et un endopodite triarticulé possède également une structure archaïque ; il faut cependant noter qu'elle n'offre que quatre endites et non cinq comme c'est le cas chez les Polyarthra et les familles les plus primitives des Oligoartha. Le maxillipède non préhensile est caractérisé par la coalescence de la précoxa et de la coxa en un coxopodite, l'endopodite étant biarticulé.

Si l'on se réfère donc aux appendices céphaliques, l'espèce de Saint-Gély-du-Fesc présente des caractères appartenant incontestablement aux Polyarthra tels ceux concernant la structure de l'antenne et de la mandibule. En revanche, la maxillule, la maxille et le maxillipède, bien que bâtis selon un type primitif, témoignent déjà de certaines réductions non moins évidentes qui empêchent de les considérer comme caractéristiques des Polyarthra.

Il convient enfin de considérer l'absence de soie au bord interne des coxa des paires de pattes natatoires et plus particulièrement au niveau de la P1. Dans le système de classification établi par Lang (1948), il s'agit là d'un caractère fondamental qui permet de séparer les Polyarthra des Oligoarthra. L'absence d'un tel caractère venant s'ajouter aux réductions minimes concernant les pièces buccales postérieures conduirait à repousser Gelyella droguei vers les Oligoarthra et plus particulièrement dans la sous-section des Maxillipedasphalea, formes chez lesquelles le premier segment thoracique est généralement libre, les maxillipèdes non encore préhensiles et les paires de pattes natatoires des mâles non transformées pour l'accouplement. L'absence de segmentation des exopodites P1, P2, P3 de la femelle et 
de l'exopodite P1 du mâle, la rudimentation des endopodites des P1, P2, P3 dans les deux sexes témoignent par ailleurs d'un phénomène de convergence assez étonnant avec le genre abyssal Cerviniella (Cerviniidae). On sait que dans ce genre, les espèces présentent le plus souvent des exopodites uniarticulés; $C$. brodskayae Por, 1969 et $C$. bodini Coull, 1973 possèdent même un endopodite $\mathrm{P} 4$ réduit à une simple protubérance ornée d'une épine. Cependant la disparition de la $\mathrm{P} 4$ et de la P5 constituent, à l'heure actuelle, un trait tout à fait original pour un Harpacticide.

En définitive, Gelyella ne possède qu'une seule des synapomorphies qui caractérise les Oligoarthra: la coxa de la P1 est dépourvue de soie interne. En revanche, elle a conservé la structure primitive de l'antenne et de la mandibule. Le schéma phylétique suivant paraît donc vraisemblable (fig. 23): Gelyella possède une souche commune avec les Oligoarthra, souche qui aurait perdu la soie interne de la coxa de P1. Mais la lignée dont elle est issue s'est isolée précocement, avant la réduction de l'antenne et de la mandibule. Au point de vue systématique, Gelyella ne peut être incluse dans aucune des familles existantes. Aussi proposons-nous la création d'une famille nouvelle, les Gelyellidae. Un remaniement plus important de la classification de Lang serait peut-être nécessaire mais sortirait du cadre de ce travail.

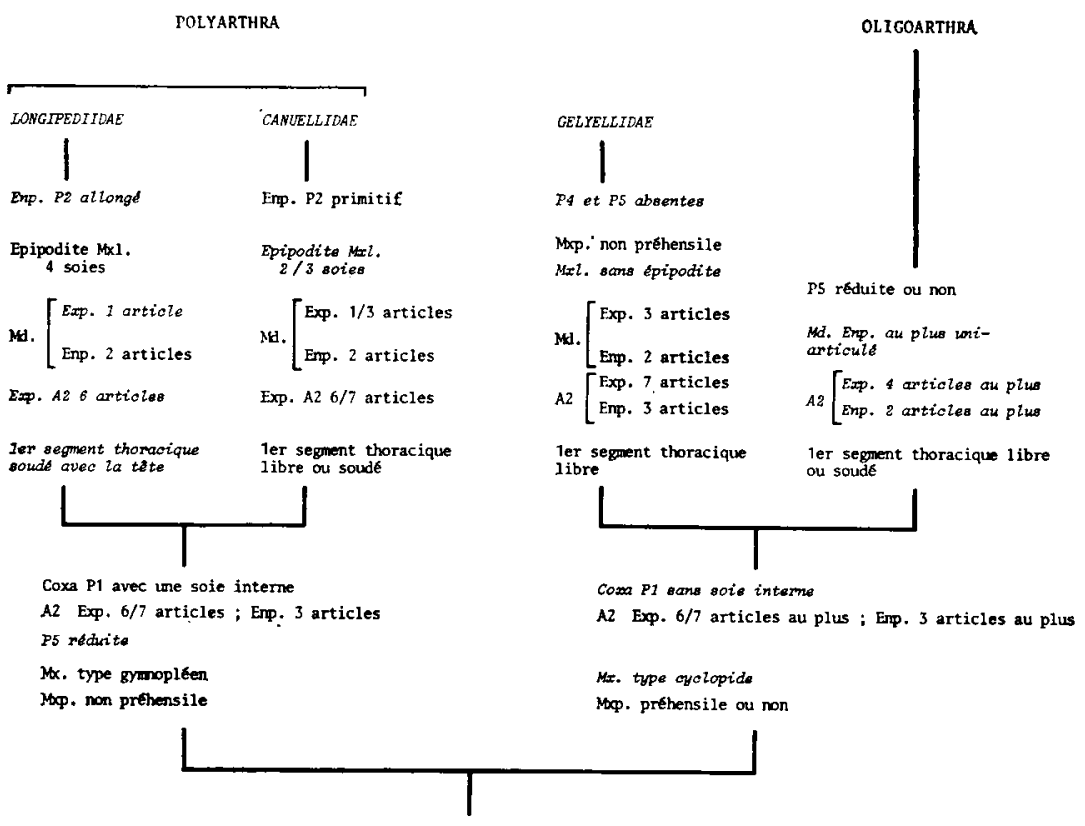

FIG. 23. - Position des Gelyellidae dans le système phylétique établi par Lang. 


\section{3. - SUR L'ORIGINE DE GELYELLA DROGUEI}

Gelyella droguei est-elle le représentant d'une vieille lignée d'eau douce aux caractères archaïques, comme c'est le cas des Phyllognathopodidae et des Chappuisidae, ou possède-t-elle, au contraire, une origine marine relativement récente? Ses caractéristiques morphologiques situent Gelyella droguei au niveau des Polyarthra et des familles les plus primitives des Oligoarthra ; ces familles sont composées, dans leur grande majorité, par des formes marines. Cependant quelques rares espèces ont également été signalées en eaux douces ou saumâtres. La découverte d'Arenosetella limnophila par Sterba en 1967, celie de Pseudectinosoma vandeli (Rouch, 1969) permettent de penser que les Ectinosomidae ont très vraisemblablement peuplé les eaux souterraines continentales sans colonisation préalable des eaux superficielles. Si l'origine de Gelyella drognei était du même type, il faudrait admettre que l'invasion des eaux souterraines continentales par des espèces marines constitue un phénomène général concernant aussi bien des formes primitives que d'autres beaucoup plus évoluées.

Cependant on trouve aussi des Oligoarthra primitifs, les Phyllognathopodidae, dans les eaux douces des cinq continents.

Seule, la découverte de nouvelles espèces de Gelyellidae pourrait permettre de préciser l'origine de cette lignée.

\section{4. - L'AQUIFÈRE KARSTIFIÉ DU LUTÉTIEN ET SON PEUPLEMENT}

L'intérêt de Gelyella droguei ne se limite pas à sa position systématique ni aux problèmes posés par son origine. Avec la capture de cette espèce, c'est un nouveau type d'aquifère karstifié de la région montpelliéraine qui s'avère riche en formes originales.

Jusqu'à ce jour, les recherches biologiques poursuivies dans les karsts du Languedoc avaient porté uniquement sur les systèmes qui se développent dans les calcaires et les dolomies du Jurassique moyen et supérieur. Ces terrains constituent en effet les aquifères les plus importants de la région et ils offrent de multiples points d'accès naturels (grottes, avens, résurgences) ou artificiels (forages). En revanche, les calcaires du Lutétien, beaucoup moins développés et peu karstifiés, ne se prêtent guère à des investigations directes. En l'absence de cavités, seules des méthodes indirectes (filtrages de résurgences ou lors de pompage d'essais) permettent de connaître leur peuplement. La découverte de Gelyella droguei incite à poursuivre les investigations dans ces formations de l'Éocène qui pourraient héberger d'autres formes originales. 


\section{REMERCIEMENTS}

Nous remercions bien vivement les Professeurs Noodt et Berthélemy ainsi que les Docteurs Schminke et Scheibel pour les précieux renseignements qu'ils ont bien voulu nous fournir.

Les figures au trait de cette note ont été exécutées par $\mathbf{M}^{\text {min' }}$ Descouens et Daffis que nous remercions chaleureusement.

\section{TRAVAUX CITÉS}

Bodin (P.). 1967. - Catalogue des nouveaux Copépodes Harpacticoïdes marins. Mém. Mus. nat. Hist. nat., 50 (1) : 11-76.

Bodin (Ph.). 1971. - Catalogue des nouveaux Copépodes Harpacticoïdes marins. Additif $\mathrm{n}^{\circ}$ 1. Tethys, 2 (4), 881-907.

Bodin (Ph.). 1976. - Catalogue des nouveaux Copépodes Harpacticoïdes marins. Additif $n^{\circ}$ 3. Univ. Bret. Occ., 1-45.

BozIc (B.). 1968. - Copépodes de la Réunion, III. Brianola curvirostris n. sp. Bull. Mus. nat. Hist. nat., 40 (3) : 570-573.

Coull (B. C.). 1971. - Meiobenthic Harpacticoida (Crustacea, Copepoda) from the North Carolina continental shelf. Cah. Biol. Mar., 12: 195-237.

Coull (B. C.). 1972. - Scottolana canadensis (Willey, 1923) (Copepoda, Harpacticoïda) redescribed from the United States East Coast. Crustaceana, 22 (3) : 209-214.

Coull (B. C.). 1973. - Meiobenthic Harpacticoida (Crustacea, Copepoda) from the deep sea of North Carolina, II. The family Cerviniidae Sars, Lang. Trans. Amer. Micros. Soc., 92 (2): 198-208.

Hamond (R.). 1973. - Four new Copepods (Crustacea: Harpacticoida, Canuellidae) simultaneously occuring with Diogenes senex (Crustacea: Paguridae) near Sydney. Proc. Linn. Soc. New'. South Wales, 97 (3) : 165-201.

Hennig (W.). 1965. - Phyllogenetic systematics. Ann. Rev. of Entomology, 10 : $97-116$.

Humes (A. G.) and Ho (J.S.). 1969. - The genus Sunaristes (Copepoda, Harpacticoida) associated with hermit crabs in the Western Indian ocean. Crustaceana, 17 (1) : 1-18.

LaNG (K.). 1948. - Monographie der Harpacticiden. Nordiska Bockandeln Stockholm.

PoR (F. D.). 1967. - Level bottom Harpacticoida (Crustacea, Copepoda) from Elat (Red sea), part 1. Isr. J. Zool., 16: 101-165.

Por (F. D.). 1969. - The Canuellidae (Copepoda, Harpacticoida) in the waters around the Sinaï peninsula and the problem of «Lessepsian" migration of this family. Isr. J. Zool., $18: 169-178$.

PoR (F. D.). 1969. - Deep-Sea Cerviniidae (Copepoda: Harpacticoida) from the western Indian Ocean, collected with RV Anton Bruun in 1964. Smithsonian Contributions to Zoology, 29: 1-60.

YoR (F. D.) and Marcus (A.). 1972. - Contribution to the knowledge of Suez canal migration. Copepoda Harpacticoidea of the Suez canal. Isr. J. Zool., $21: 249-274$.

Roucii (R.). 1969. - Recherches sur les eaux souterraines, 6. Sigmatidium vandeli n. sp., Ectinosomidae des eaux souterraines continentales. Ann. Spéléol., 4 (2) : 421-429.

SoYer (J.). 1966. - Copépodes Harpacticoïdes de Banyuls-sur-Mer, 3. Quelques formes du coralligène. Vie et Milieu, 17 (1-B) : 303-344.

Sterba (O.). 1967. - K Poznani Plazivek (Copepoda, Harpacticoidea) Asyske casti Plearktidy. Acta Univ. Palackianae Olomucencis, Fac. Rer. Nat., 25 : 251-380. 